\title{
The expert in hemostasis and thrombosis in the Italian health system: role and requirements for a specific clinical and laboratory expertise
}

\author{
Francesco Rodeghiero, ${ }^{1}$ Massimo Morfini, ${ }^{2}$ Carlo Nozzoli, ${ }^{3}$ Cesare Manotti, ${ }^{4}$ Francesco Violi, ${ }^{5}$ Giuseppe Castaldo, ${ }^{6}$ \\ Fabrizio Pane, ${ }^{7}$ Bruno Biasioli, ${ }^{8}$ Claudio Velati ${ }^{9}$ on behalf of SISET, AICE, FADOI, FCSA, SIBioC, SIE, SIMeL, \\ SIMI, SIMTI
}

\begin{abstract}
${ }^{1}$ Dipartimento di Terapie Cellulari ed Ematologia, Ospedale San Bortolo, Vicenza, on behalf of SISET; ${ }^{2}$ AOU Careggi, Firenze, on behalf of AICE; ${ }^{3}$ Dipartimento Area Medica, AOU Careggi, Firenze, on behalf of FADOI; ${ }^{4}$ AUSL Parma, Centro per le Malattie dell'Emostasi e cura dell'Emofilia, Parma, on behalf of FCSA; ${ }^{5}$ Divisione di Medicina Interna C, Università La Sapienza, Policlinico Umberto I, Roma, on behalf of SIMI; ' Dipartimento di Biochimica Clinica e Biotecnologie Mediche, Università di Napoli Federico II, on behalf of SIBioC; ${ }^{7}$ UO Ematologia e Trapianti di Midollo, AOU Federico II, Napoli, on behalf of SIE; ${ }^{8}$ Ospedale Maggiore - AOU Ospedali Riuniti, Trieste, on behalf of SIMeL; ${ }^{9}$ Servizio di Immunoematologia e Medicina Trasfusionale, AO Valtellina e Valchiavenna, on behalf of SIMTI
\end{abstract}

\begin{abstract}
Hemorrhagic and thrombotic diseases are highly heterogeneous disorders that may affect a large proportion of the population, as in the case of patients taking antithrombotic drugs. The appropriate management of such conditions requires the availability of specific diagnostic assays, together with knowledge of the possible clinical syndromes and of their appropriate treatment. This can only be achieved through second-level specialized laboratories supervised by trained personnel. Such diagnostic and therapeutic organization is not widely available in Italy except in a very limited number of those large hospitals that are centers of excellence on a national scale. Increasing the availability of such resources would be of great benefit to patients, and could also be cost-effective for the national healthcare system. This document is promoted by the Italian Society for the Study on Hemostasis and Thrombosis (SISET) and by the main Italian scientific societies involved in the field during the years 2011-2012. It aims to identify the level of scientific and professional training required to define a physician as a Hemostasis and Thrombosis Expert, graded according to the levels of skill required for different clinical settings.
\end{abstract}

Correspondence: Francesco Rodeghiero, Dipartimento di Terapie Cellulari ed Ematologia, Ospedale San Bortolo, viale Rodolfi 37, 36100 Vicenza, Italy.

Tel.: +39.0444.753626 - Fax: +39.0444.753365.

E-mail: rodeghiero@hemato.ven.it

Key words: blood coagulation disorders, laboratory diagnosis, treatment, epidemiology.

Conference presentation: this document was presented at the XXII Congress of the Italian Society on Hemostasis and Thrombosis (Società Italiana per lo Studio dell'Emostasi e della Trombosis, SISET) held in Vicenza, Italy on 4-6 October, 2012.

Received for publication: 27 June 2013.

Revision received: Not required.

Accepted for publication: 22 July 2013.

This work is licensed under a Creative Commons Attribution NonCommercial 3.0 License (CC BY-NC 3.0).

CCopyright F. Rodeghiero et al., 2013

Licensee PAGEPress, Italy

Italian Journal of Medicine 2013; 7(s8):71-73

doi:10.4081/itjm.2013.s8.71

\section{Introduction}

This paper identifies the scientific knowledge and the clinical and laboratory skills required by a physician or by other medical graduate in charge of clinical and/or laboratory activities to fully accomplish his or her duties according to the professional standard required in the field of hemostasis and thrombosis. A person with this professional profile is called an $E x$ pert in Hemostasis and Thrombosis (EHT). We summarize the consensus reached by the main Italian Scientific Societies in this field. The paper has been coordinated by the Chairman of the Italian Society for the Study on Hemostasis and Thrombosis (SISET) during his 2011-2012 tenure.

The main tenet underlying this document is the awareness that, in Italy, clinical needs in terms of diagnosis, treatment and prevention of bleeding and thrombotic disorders are not adequately met by the currently available inpatient and outpatient services. This inadequacy is mainly due to a lack of sufficient numbers of EHTs, by the lack of awareness of their critical role within hospitals, and by the limited availability of specific post-graduate academic training. 


\section{Epidemiology of thrombotic and hemorrhagic disorders}

Consideration of the epidemiology of thrombotic and hemorrhagic disorders helps us to fully appreciate the relevance of this proposal. These disorders are congenital or inherited, and encompass a wide spectrum of severity affecting all ages. They also affect pregnant women and newborns or toddlers, in whom they present with distinctive features requiring a specific approach. Severe congenital coagulopathies, such as hemophilias or thrombophilia, or acquired bleeding/thrombotic manifestations secondary to drug treatment (heparin-induced thrombocytopenia, hemorrhages secondary to anticoagulation) may require treatment in well-established centers. On the other hand, the expertise to suspect or identify these disorders should be more widely distributed. Prevention of venous and arterial thrombosis involves a vast number of the population and, although not usually dealt with by the EHT, he is often consulted in cases with critical comorbidities. Furthermore, EHTs are among the main players in preparing national or local guidelines or protocols in the general field of hemostasis and thrombosis.

\section{General skills}

An integral approach to the patient requires that the attending physician receive a comprehensive education and training in internal medicine, followed by a more specific specialization in the various disorders of hemostasis and thrombosis, as described below.

As far as laboratory medicine is concerned, investigation of hemostatic disorders requires the availability of specific diagnostic assays together with knowledge of the possible clinical syndromes and/or their appropriate treatment. This can only be achieved through second-level specialized laboratories supervised by appropriately trained medical graduates.

The minimum standard of specific knowledge and skills that should characterize the EHT have been identified as follows: i) knowledge of the physiopathology of hemostasis; ii) knowledge of the classification and epidemiology of the congenital and acquired hemostatic disorders; iii) knowledge of the molecular basis of hemostatic disorders; iv) ability to diagnose the main hemorrhagic and thrombotic disorders, and the ability to at least suspect even the more rare diseases in order to refer these patients to tertiary referral centers. These skills should be based on a rational systematic approach in which patients' personal and familial history and physical examination are integrated with the results of clinical and laboratory investigations; v) knowledge of the principles governing the specific (both phenotypic or genotypic) laboratory tests, and of their clinical utility; vi) technical competence in establishing or at least supervising the execution of the laboratory tests; vii) knowledge of the scientific methodology required for a critical interpretation of clinical trials, meta-analysis, systematic review of literature, and of the principles and application of health technology assessment.

Hemorrhagic and thrombotic complications in vital organs such as myocardium, brain, gastrointestinal tract are best managed by organ specialists (cardiologist, neurologist, surgeons, etc.) consulting the EHT when required.

\section{Specific skills and abilities required in the different healthcare contexts}

The EHT should usually be specialized in internal medicine or in hematology. He or she should have completed adequate clinical training in the general aspects of medicine, complemented by a specific experience in hemostasis and thrombosis. This training can be integrated during the post-graduate course of internal medicine or hematology specialties. However, the current curricula should be complemented by a more extensive education and training in the pertinent sectors for hematology and, in particular, for internal medicine specialties, and by a more extensive education and training in the general aspects of internal medicine for hematologists. In either case, skills training in laboratory diagnosis must be completed. Clinical biochemistry or laboratory medicine specialties should also revise their curriculum by giving more attention to hemostasis and thrombosis.

The European Hematology Curriculum has provided a sound base on which to categorize the skills required by the general hematologist and these can be used as a framework to grade skills for the EHT, as may be required in the different clinical and laboratory settings (http://www.ehaweb.org/assets/documents/ CV-PASSPORT-2012.pdf).

Two main aspects can be considered, the first concerning patient management, the second concerning laboratory skills and laboratory-assisted diagnosis. For each of these, 3 levels of education, skill and clinical competence can be proposed: i) the ability to correctly characterize the patient's condition and to describe the general range of laboratory tests required; ii) the ability to start a diagnostic or therapeutic process using established treatment protocols and to correctly identify referral routes, particularly for rare conditions; the capability of identifying the need for and to establish urgent consultation; full understanding of the main laboratory tests; iii) the ability for independent decision making as to the management and/or treatment (and monitoring of treatment) for bleeding and hemorrhagic 
disorders, with the help of specific laboratory tests if required.

To qualify as an EHT, a medical graduate should have at least Level 2 competence in both patient management and laboratory diagnosis. Level 3 is required for an autonomous management of patients. Further information is available in the 2013 inter-society dossier. ${ }^{1}$

\section{Post-gradute training in Italy}

On a European level, hemostasis and thrombosis is emerging as an autonomous discipline for which a specific academic specialty is not available. In Italy, it seems that an integration of the curriculum of hematology and of internal medicine specialties with dedicated education and training in the final 2-3 years of study would represent a feasible and efficient way to cover the needs of EHTs in future years.

A strict collaboration between the EHT and medical specialists, and/or biologists/scientists working in dedicated laboratories is mandatory. This implies that also these personnel should receive a comprehensive education and training in hemostasis and thrombosis. This could initially be accomplished by integrating the curricula of clinical biochemistry or laboratory medicine specialties.

\section{The role of the Expert in Hemostasis and Thrombosis in the Italian healthcare system}

Four levels of complexity are identified: i) smallmedium sized hospitals, serving less than 200,000 people, or even smaller hospitals in an isolated area: at least 1 EHT with at least Level 2 competence should be present or available. Routine diagnostic laboratory facilities should be made available for coagulation screening and oral anticoagulation monitoring; ii) medium-sized hospitals, serving 200,000-600,000 people: a dedicated EHT specialist with Level 3 competence should be present on site. Laboratory services dedicated to hemostasis and thrombosis should also be available; iii) referral hospitals serving more than 600,000 people should have a center dedicated to hemostasis and thrombosis. The director of the center should have
Level 3 competence. Additional personnel, including other EHTs of at least Level 2, and a dedicated laboratory should be available. This laboratory could be under the supervision of the director of the center or under the supervision of a laboratory internal medicine expert with Level 3 competence; iv) national referral centers for hemostasis and thrombosis.

Efforts should be made to formally establish a network between all these different services, assuring round the clock availability or consultation with a Level 3 EHT and 24-h accessibility to a dedicated laboratory for urgent coagulation testing (e.g. coagulation inhibitors, heparin-induced thrombocytopenia testing, monitoring of new oral anticoagulants, etc.).

\section{Conclusions}

This inter-society document has received the full approval of the signatory societies. It is strongly believed that implementation of the suggested measures will have a positive impact on the Italian healthcare system by improving diagnosis and treatment and consultation services in hemorrhagic and thrombotic disorders, and will also avoid the unnecessary risks associated with antihemorrhagic or antithrombotic treatments.

\section{Acknowledgments}

We would like to thank the Chairmen of the various signatory societies. We would also like to thank the following for their critical contribution in drafting and revising the Italian version of this document: Pasquale Pignatelli (SIMI), Mario Plebani (SIBioC), Angiola Rocino (AICE), Gina Rossetti (SIMTI), Mauro Silingardi and Giorgio Vescovo (FADOI), Sophie Testa (SIMeL), Alberto Tosetto (FCSA).

\section{References}

1. Rodeghiero F, Morfini M, Nozzoli C, et al. Identificazione e ruolo dell'esperto in emostasi e trombosi nel sistema sanitario nazionale italiano. Documento intersocietario a cura di SISET, AICE, FADOI, FCSA, SIBioC, SIE, SIMeL, SIMI, SIMTI. Riv Ital Med Lab 2013;9:134-42. 